\title{
Midpoint vs Single Score in Multi-Criteria Optimization under Life Cycle Assessment Constraints: the Case of Potable Water Treatment Chains
}

\author{
Florin Capitanescu, Elorri Igos, Antonino Marvuglia, Enrico Benetto \\ Environmental Research and Innovation (ERIN) \\ Luxembourg Institute of Science and Technology (LIST) \\ 41, rue du Brill, L-4422 Belvaux, Luxembourg \\ \{florin.capitanescu,elorri.igos,antonino.marvuglia,enrico.benetto\}@list.lu
}

\begin{abstract}
This paper addresses the multi-objective constrained optimization of a drinking water production plant. It reports the successful coupling between the Strength Pareto Evolutionary Algorithm (SPEA2), which is a well-established multi-goal elitist metaheuristic global optimizer, and EVALEAU, which is a state-of-the-art process modelling - life cycle assessment (LCA) tool for simulation of potable water treatment chains. The paper assesses as well the pros and cons of using midpoint versus single score as environmental goal metrics in a decision making process based on very challenging multi-goal optimization of potable water treatment chains. The proposed approach is illustrated using the model of a real-world drinking water production plant.
\end{abstract}

Keywords- drinking water production plant; life cycle assessment; multi-objective optimization; meta-heuristic algorithms; ReCiPe single score; ReCiPe midpoint level impact.

\section{INTRODUCTION}

Life cycle assessment (LCA) is a standardized methodology which analyzes the environmental performance of any industrial process during its entire lifecycle [1]. In order to offer decision makers efficient solutions for reducing the environmental impact of a process, LCA needs to be complemented by multi-goal optimization tools.

The extension of industrial process optimization to further account for environmental constraints has been broadly investigated so far [2]. Most of the previous works assume that the process can be modeled analytically by a set of equations enabling thereby resorting to suitable mature mathematical programming-based methods [3],[4],[5]. However, the detailed simulation of water treatment chains involves a deep level of software embedding which precludes the use of classical derivative-based mathematical programming methods and hence calls for meta-heuristic algorithms [6] which are suitable for such black-box model multi-goal optimization. This is the case of the EVALEAU simulator, which is a state-of-the-art flowsheet-based process modelling - LCA tool for prospective and retrospective simulation of potable water treatment chains [7]. EVALEAU was developed in Umberto ${ }^{\circledR}$, relies on the software PHREEQC® [8] for water chemistry calculation, and its modules are linked to the Ecoinvent ${ }^{\circledR}$ database [9] for the LCI of background processes. The EVALEAU library is composed of a certain number of unit processes for water treatment, which can be further combined to simulate a specific treatment chain, like e.g. the one in Fig. 1.

On the other hand, comparatively with the previous works on LCA-based optimization, the optimization of potable water production plants taking into account the lifecycle impact has received very little attention up to now. The only existing work [10] performs a single-objective optimization of a water production plant by combining the Nelder-Mead local search algorithm and EVALEAU. However, it does not consider multiple objectives or water quality constraints, and the local search algorithm utilized may be trapped into local optima.

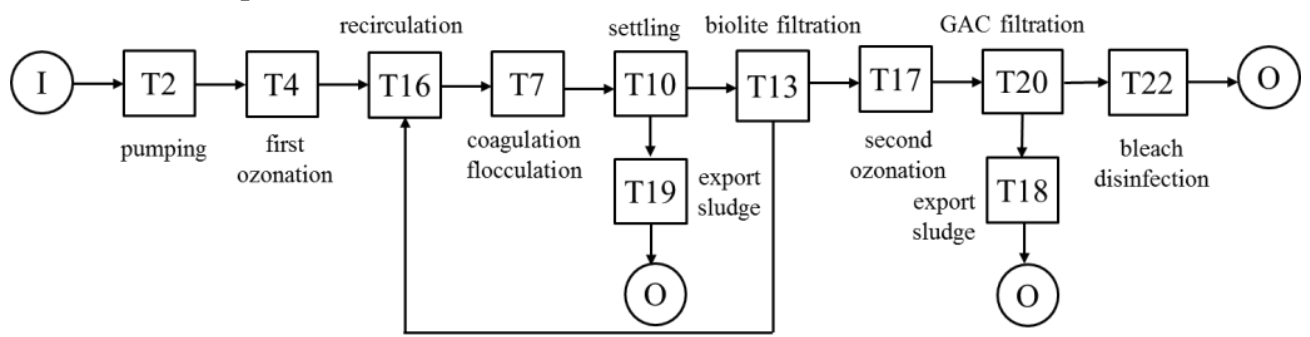

Figure 1: Simplified model of the drinking water production plant used in the case study. "I" stands for "input" and "O" stands for "output". 
Aiming to mitigate these drawbacks, a first contribution of this paper is the successful coupling between a multi-objective global optimization tool and EVALEAU. Key design operational parameters of the potable water plant treatment chain are optimized by resorting to a metaheuristic global optimization algorithm, called Strength Pareto Evolutionary Algorithm (SPEA2) [11], which is a natural candidate for black-box multi-objective optimization.

Furthermore, another contribution of the paper is the evaluation of the pros and cons of using midpoint versus single score as environmental goal metrics in a decision making process based on very challenging multi-goal optimization of drinking water treatment chains.

The remaining of the paper is organized as follows. Section II states conceptually the optimization problem, describes the coupling approach and presents the global optimizer used. Section III discusses the pros and cons of various environmental metrics. Section IV provides optimization results with the SPEA2 algorithm for a model of a real-world potable water production plant. Section V concludes.

\section{OVERVIEW OF THE APPROACH COUPLING A GLOBAL MULTI- OBJECTIVE OPTIMIZER AND EVALEAU SIMULATOR}

\section{A. Abstract Problem Statement}

The basic multi-goal optimization problem in the context of potable water treatment plant can be formulated conceptually by the set of equations (1)-(4):

$\min \left\{f_{1}(\mathbf{x}), f_{2}(\mathbf{x}), f_{3}(\mathbf{x}), \ldots\right\}$

subject to:

$g(\mathbf{x})=0$

$h_{\min }<=h(\mathbf{x})<=h_{\max }$

$\mathbf{x}_{\min }<=\mathbf{x}<=\mathbf{x}_{\max }$

The problem consists in finding optimal settings for some key decision variables, denoted by $\mathbf{x}$, according to the trade-off between conflicting objectives such as the operational cost, denoted by $f_{1}(\mathbf{x})$, and some environmental impact metrics, denoted by $f_{2}(\mathbf{x}), f_{3}(\mathbf{x}), \ldots$, etc. The optimization problem satisfies a set of constraints on the process unit input-output mass-flow balance (2), the output water quality (3) and design parameters physical range (4).

In the above problem formulation the environmental impact metrics are calculated by the widely used ReCiPe Life Cycle Impact Assessment (LCIA) method [12], which is applied at either midpoint or endpoint levels.

The water potability is enforced by constraints (3), focusing, at water production plant experts suggestion, on seven major aggregated parameters (e.g. total coliforms, total trihalomethanes, total organic carbon, Escherichia coli, faecal streptococci, turbidity, and conductivity).

\section{B. Proposed Approach and Software Architecture}

The proposed approach for multi-objective constrained optimization of the drinking water production plant is shown in Fig. 2 and consists in linking the EVALEAU simulator with the multi-objective global optimizer SPEA2. The advantage of this solution is that the optimization problem (1)-(4) is decomposed in two convenient blocks which are solved sequentially: (i) solution of equality constraints (2) obtained by running the EVALEAU simulator for a given value of decision variables $\mathbf{x}$ provided by the optimizer, and (ii) improvement of the current set of solutions of the optimization problem (1)-(4) by the global optimizer, which receives as input the values of functions $f_{1}(\mathbf{x}), f_{2}(\mathbf{x})$, and $h(\mathbf{x})$ already evaluated by the EVALEAU simulator, returning to the latter a new promising value of decision variables $\mathrm{x}$. The communication between EVALEAU simulator and SPEA2 optimizer is steered in Umberto ${ }^{\circledR}$ via a Python script, the automated communication with the simulator relying on the advanced-user functions which are available in Umberto®.

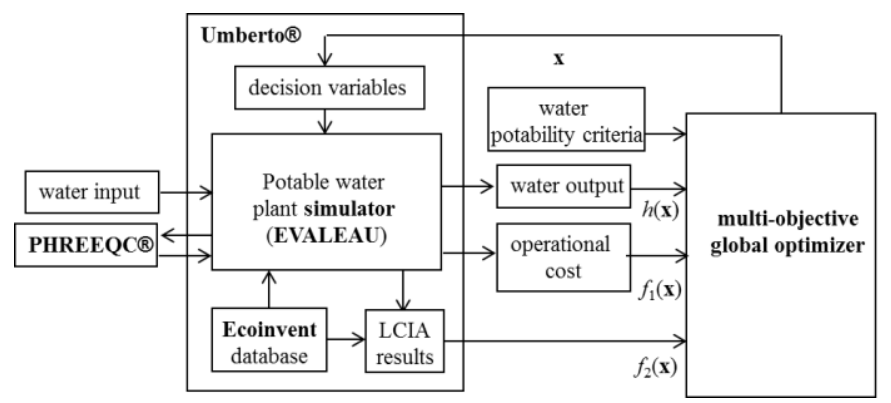

Figure 2: High level software architecture of the coupling between a multi-objective global optimizer and EVALEAU simulator.

SPEA2 [11] is a well-established elitist global evolutionary meta-heuristic derivative-free multi-objective optimizer which has been successfully applied to many black-box optimization problems in various engineering fields. It aims at finding or approximating the optimal set of trade-off solutions (i.e. the Pareto front). As any evolutionary algorithm, once an initial population is randomly generated, it repeats the following steps: evaluation, selection, crossover and mutation, until some termination criteria are met (e.g., generally a pre-defined number of generations is reached). SPEA2 is an improved version of the initial SPEA algorithm, incorporating, as described in [11], additional features such as a fine-grained fitness assignment strategy (which takes into account for an individual the numbers of individuals it dominates and is dominated by), a nearest neighbor based density estimation technique, and an enhanced archive truncation method which guarantees the preservation of boundary solutions. SPEA2 operates with a population (archive) of fixed size, from which promising candidates are drawn as parents of the next generation. The resulting offspring then compete with the old ones for inclusion in the population.

SPEA2 algorithm is provided as free source code on the PISA platform [13] and has been adapted to the optimization problem addressed in this paper. 


\section{PROS AND CONS OF ENVIRONMENTAL METRICS FOR THE WATER PLANT OPTIMIZATION PROBLEM}

We briefly discuss the pros and cons of two environmental metrics.

Using ReCiPe single score indicator [12] as single environmental impact metric leads to a bi-objective optimization problem. This approach has two advantages: it facilitates decision making as the analysis is reduced to a twodimensional set of optimal solutions (i.e. the Pareto front), and the computational effort, although very significant, is the least one can expect for this type of multi-objective optimization problem. However, the decision making relies on questionable assumptions used to compute the single score, and may hide the effect of potentially significant impact categories due to the conversion to endpoints and to the arbitrary weights assigned in aggregation.

Using some ReCiPe midpoint level impact categories [12] as independent environmental goals in the multi-objective optimization problem has the major advantage of reliance upon relatively widely accepted methods and indicators. However, the computational complexity (i.e. approximation of Pareto front and running time) of the multi-objective optimization problem grows dramatically with the number of conflicting objectives and may become unmanageable [14]. This critical issue gets even worse for time consuming simulators as EVALEAU, which takes around 180 seconds per simulation on a $2.70 \mathrm{GHz} / 8 \mathrm{~GB}$ computer. Furthermore, for the optimization of more than three independent objectives the visual interpretability of the Pareto front becomes problematic. Hence, decision maker-based careful reduction of the number of conflicting environmental goals to be optimized is desirable. This may benefit from the fact that some impact categories have the same trend while other environmental impacts of interest can be handled as constraints (but the optimization results may depend on their beforehand expert-based limits).

\section{NUMERICAL EXPERIMENTS}

\section{A. General Assumptions}

The proposed approach is illustrated using a realistic model of an existing French drinking water production plant shown in Fig. 1. The EVALEAU simulator runs in Umberto® 5.6 environment and is linked to the Ecoinvent ${ }^{\circledR}$ v2.2 LCI database for background processes. The environmental impact objectives are computed using the ReCiPe LCIA method. The functional unit is $1 \mathrm{~m}^{3}$ of potable water produced while monetary units (m.u.) stand for euro.

We focus on optimizing the settings of the six most efficient design variables: the ozone transfer efficiency and the pure oxygen fraction in feed gas in both $\mathrm{T} 4$ and $\mathrm{T} 17$ ozonation unit processes (see Fig. 1), the coagulant dose in the coagulation/flocculation T7, and the granular activated carbon (GAC) regeneration frequency in the GAC filtration T20.

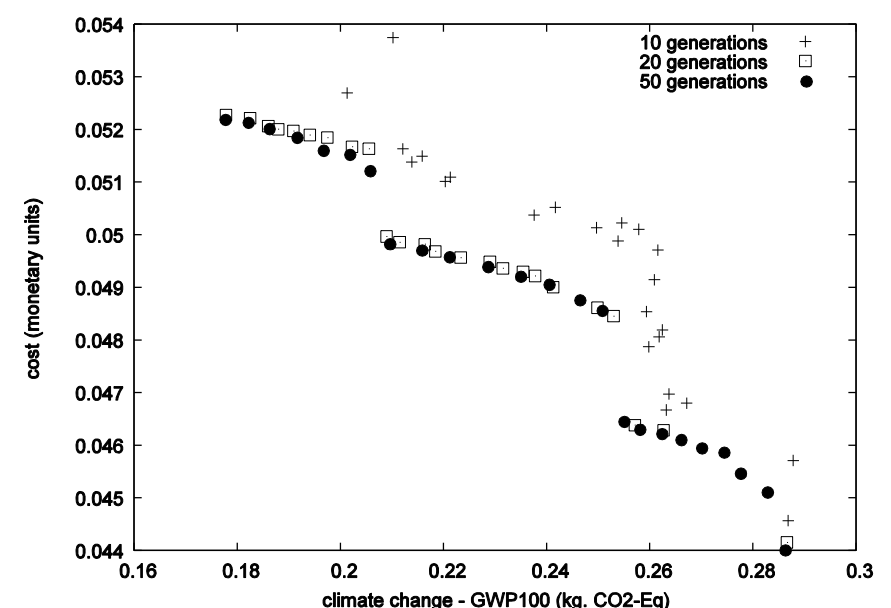

Figure 3: SPEA2 convergence over generations: cost vs. midpoint GWP100 environmental metric.

In the following experiments, without otherwise specified, the SPEA2 algorithm is run with default settings for a population size of 24 individuals during 50 generations (as the algorithm practically converged to the Pareto front).

\section{B. Results using SPEA2 Algorithm}

Figure 3 shows the 2D Pareto front trading-off cost vs. environmental impact (focusing only on climate change impact category by means of the Global Warming Potential GWP - factors based on 100 years-time horizon). One can see that the Pareto front is composed of three disjoint concave parts. Note that most trade-off optimal solutions allow a reduction of both objectives compared to the initial drinking water production plant operating point, which corresponds to a cost of 0.0578 monetary units (m.u.) and the GWP100 environmental impact of $0.2158 \mathrm{~kg} \mathrm{CO}$-Eq. In particular the solution with the lowest environmental impact leads to a reduction of the cost and the environmental impact of $9.7 \%$ and $18.0 \%$, respectively.

The figure also provides three snapshots of the front approximation during generations. As expected after only 10 generations the front approximation is still far from the Pareto front and several solutions are dominated points. Then, the front approximation after 20 generations contains only nondominated solutions and approaches very closely the Pareto front. However, after 20 generations the solutions spread is still insufficient, see e.g. a small number of points in the right part of the figure.

Note that the computational effort of the algorithm corresponds mainly to $24 \times 50=1200$ runs of EVALEAU and takes around 60 hours. This computational burden supports the a priori identification of the most effective decision variables. Although this computational effort is not a major concern at the planning stage, further speed-up of both the optimizer and EVALEAU tool are welcome. 


\section{Midpoint vs Single Score}

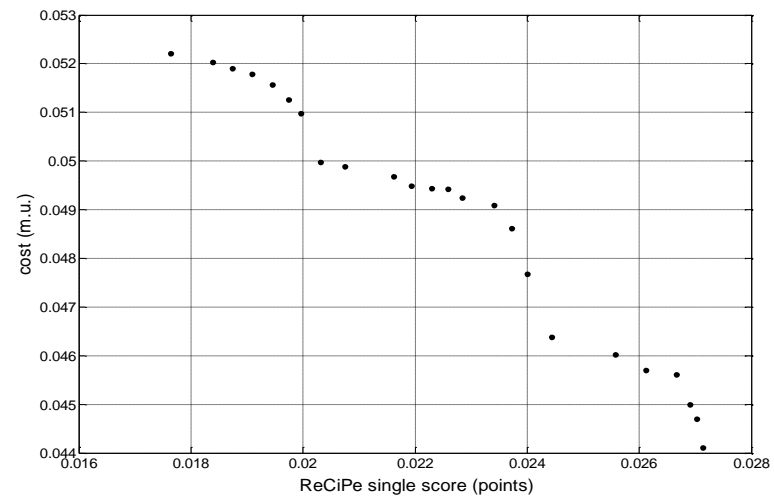

Figure 4: Pareto front: cost vs. single score indicator.

Figure 4 shows the $2 \mathrm{D}$ cost vs. single score optimal tradeoff Pareto front obtained with the SPEA2 algorithm when the single score is chosen as environmental metric. The single score indicator aggregates three impact categories, namely "ecosystem quality", "human health", and "resources" assigning them the following weights: $40 \%, 40 \%$, and $20 \%$, respectively. Figures 5 and 6 display LCA results for these impact categories, both overall and individually, specifically for the Pareto point in Fig. 4 which corresponds to the lowest environmental impact and largest cost.

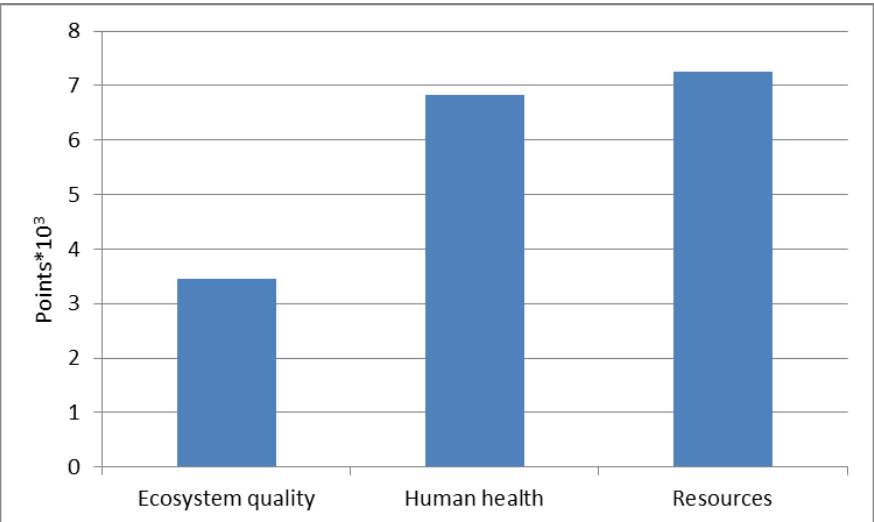

Figure 5: Single score overall impact per category for the point of the Pareto front with the lowest environmental impact.

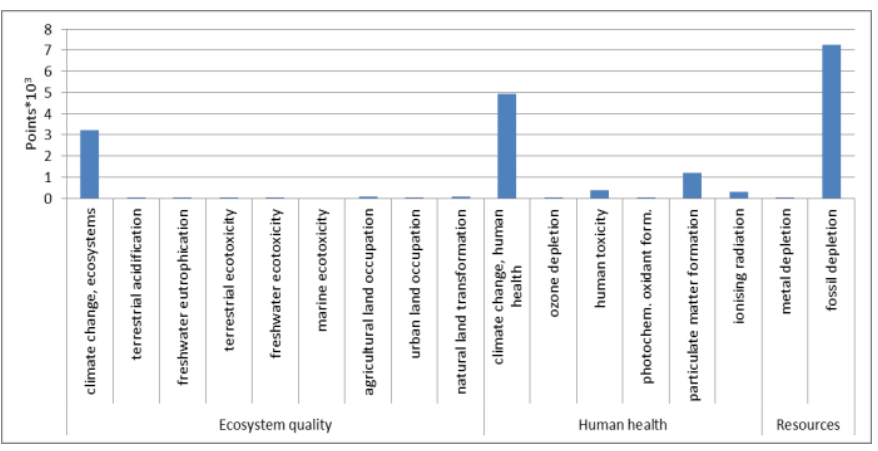

Figure 6: Single score impact detail per each midpoint impact category for the point of the Pareto front with the lowest environmental impact.

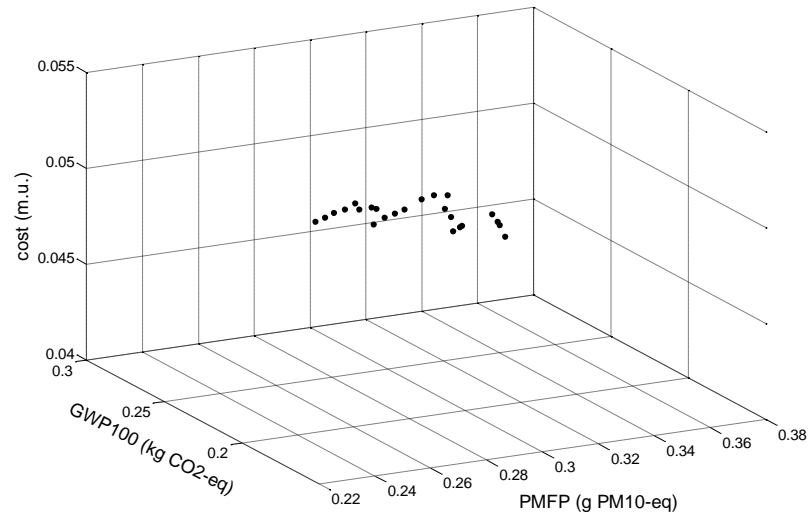

Figure 7: Pareto front: cost vs. climate change vs. particulate matter formation potential.

Figure 7 shows the 3D cost vs. climate change vs. particulate matter formation potential trade-off Pareto front obtained with the SPEA2 algorithm when two midpoint level environmental goal metrics are considered. The two midpoint categories were chosen because of their relevance in the LCA of water treatment chains as well as due to their relatively more important impact (see Fig. 6).

Looking closely at Figs. 3, 4 and 7 one can note that the Pareto fronts of single score and mid-point have quite similar shapes. This is due to the significant weight of the climate change in the single score (it counts overall for $46 \%$, see Fig. 6) as well as the common trend for most impact categories (e.g. the graph in Fig. 7 shows indeed that GWP100 and PMFP metrics are not in conflict).

\section{Impact of the Population Size in SPEA2}

We now run SPEA2 using a small population of 6 individuals, like in a micro-genetic algorithm approaches.

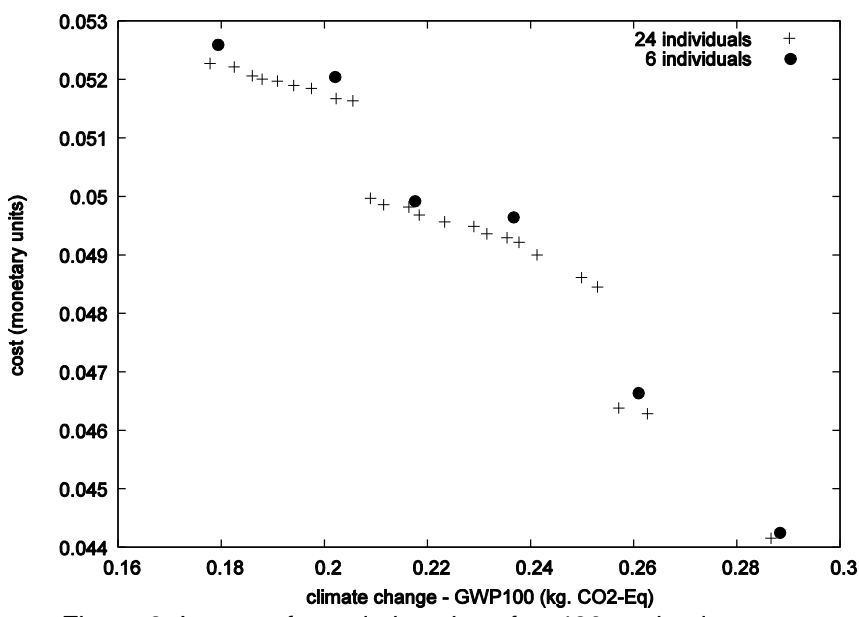

Figure 8: Impact of population size after 480 evaluations.

Figure 8 provides the results obtained with SPEA2 algorithm after 480 evaluations with both population sizes 6 and 24 respectively. While one would expect that the smaller the population size the faster the convergence to the Pareto front, the figure shows that the contrary holds, i.e. as the population gets larger, it not only covers better (evenly) the 
whole Pareto front, but also slightly dominates the small size population in the objective space.

Finally we run SPEA2 with a population of 24 individuals but allowing only 12 parents to breed 12 children, instead of the default option which replaces the whole population (i.e. 24/24 option). Figure 9 shows that, compared with the default option, the 12/12 option fails in this case and after 480 evaluations to explore the upper part of the Pareto front.

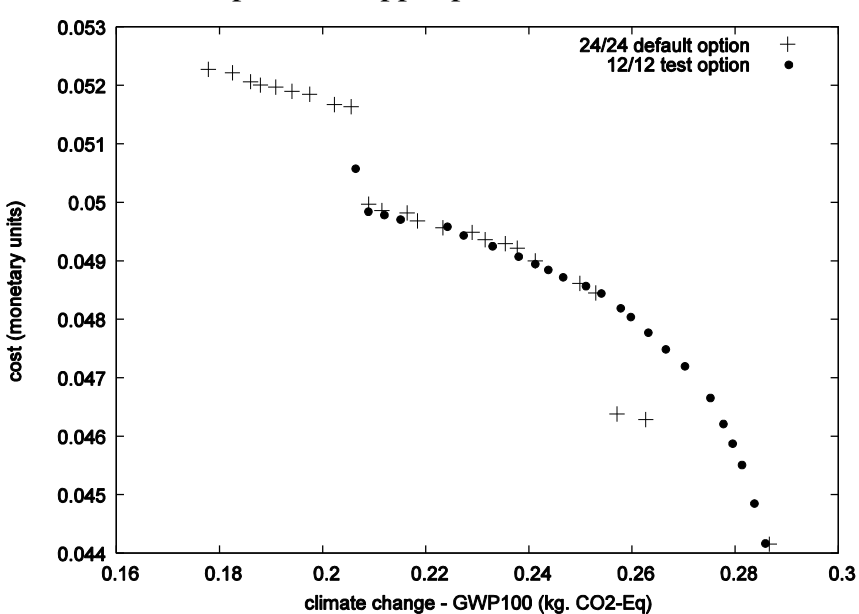

Figure 9: Impact of population replacement rate.

\section{CONCLUSIONS}

This work demonstrates the successful application of the global optimizer SPEA2 in combination with the process engineering - LCA simulator to provide optimal settings of design variables, according to the trade-off cost vs. environmental impacts, for a potable water production plant.

Multi-goal optimizers could be easily combined to other LCA simulators targeting other industrial sectors.

This work further offers a perspective of the approaches based on midpoint indicators versus those based on single score indicator for decision making from the viewpoint of challenging multi-goal optimization problems over computationally expensive "process modeling - LCA" simulators of potable water treatment chains. The results show that solving such optimization problems under both approaches is technically feasible provided that, for midpoint approach, a small number of key environmental impact metrics are carefully chosen. In the presence of adequate information, other midpoint impact categories can be straightforwardly incorporated into the optimization problem as constraints.

Future work will explore the potential reduction of the computational burden of the algorithm for expensive blackbox optimization problems, e.g. by using hybrid algorithms combining suitable global and local search methods or relying on surrogate black-box models.

\section{ACKNOWLEDGEMENT}

The authors acknowledge the funding from Luxembourg Research Fund (FNR) in the frame of OASIS project
(CR13/SR/5871061). The authors wish to thank Prof. Ligia Tiruta-Barna (INSA Toulouse) for the valuable support regarding the realistic simulation of the water treatment chain and Dr. Aras Ahmadi (INSA Toulouse) for the data provided and support in setting-up the multi-objective constrained optimization problem.

\section{REFERENCES}

[1] ISO 14040: Environmental Management - Life Cycle Assessment Principles and Framework, (2006), International Organisation for Standardisation, Geneva (Switzerland).

[2] L. Jacquemin, P.-Y. Pontalier, C. Sablayrolles, "Life Cycle Assessment (LCA) Applied to the Process Industry: A Review", Int. J. Life Cycle Assess., 17, 2012, pp. 1028-1041.

[3] A. Azapagic, R. Clift, "Life Cycle Assessment and Multiobjective Optimisation”, J. Clean. Prod., 7, 1999, pp. 135-143.

[4] B.H. Gebreslassie, G. Guillén-Gosálbez, L. Jiménez, and D. Boer, "Design of environmentally conscious absorption cooling systems via multi-objective optimization and life cycle assessment", Applied Energy, 86 (9), 2009, pp. 1712-1722.

[5] M. Schmidt, H. Lambrecht, and A. Möller, "Optimisation approaches in material flow models of manufacturing systems", Environmental Informatics and Systems Research conference, Warsaw, Poland, 2007.

[6] Y. Méry, L. Tiruta-Bana, E. Benetto, I. Baudin, “An Integrated "Process Modelling-Life Cycle Assessment" Tool for the Assessment and Design of Water Treatment Processes", Int. J. Life Cycle Assess. 18, 2013, pp. 1062-1070.

[7] A. Zhou, B.-Y. Qu, H. Li, S.-Z. Zhao, P.N. Suganthan, Q. Zhang, "Multiobjective evolutionary algorithms: A survey of the state of the art", Swarm and Evolutionary Computation, 1 , 2011, pp. 32-49.

[8] D.L. Parkhurst, C.A.J. Appelo, "Description of input and examples for PHREEQC version 3--A computer program for speciation, batchreaction, one-dimensional transport, and inverse geochemical calculations", U.S. Geological Survey Techniques and Methods, book 6 , chap. A43, 2013, 497 p.

[9] B.P. Weidema, C. Bauer, R. Hischier, C. Mutel, T. Nemecek, J Reinhard, G. Wernet, "Overview and methodology: Data quality guideline for the ecoinvent database version 3", Swiss Centre for Life Cycle Inventories, 2013.

[10] R.J. Wallace, A. Marvuglia, E. Benetto. L. Tiruta-Barna, "A New Approach to Optimization with Life Cycle Assessment: Combining Optimization with Detailed Process Simulation", ICCSA 2014 (Eds. Murgante B. et al.), LNCS, 8581, 2014, pp. 707-720.

[11] E. Zitzler, M. Laumanns, L. Thiele, "SPEA2: Improving the Strength Pareto Evolutionary Algorithm for Multiobjective Optimization," Evolutionary Methods for Design, Optimisation and Control with Application to Industrial Problems. Proceedings of the EUROGEN2001 Conference, Greece, 2001, pp. 95-100.

[12] M.J. Goedkoop, R. Heijungs, M. Huijbregts, A. De Schryver, J. Struijs, R. Van Zelm, "ReCiPe 2008, A life cycle impact assessment method which comprises harmonised category indicators at the midpoint and the endpoint level, First edition Report I: Characterisation,” 6 January 2009, http://www.lcia-recipe.net.

[13] S. Bleuler, M. Laumanns, L. Thiele, E. and Zitzler, "PISA - A Platform and Programming Language Independent Interface for Search Algorithms", In: Fonseca C.K., Fleming P.J., Zitzler E., Deb K. and Thiele L. (Eds.), Evolutionary Multi-Criterion Opti-mization (EMO 2003), LNCS, Berlin, Springer, 494-508, 2003.

[14] D. Brockhoff, E. Zitzler, “Are All Objectives Necessary? On Dimensionality Reduction in Evolutionary Multiobjective Optimization", in T. P. Runarsson et al., editors, Conference on Parallel Problem Solving from Nature (PPSN IX), volume 4193 of LNCS, pp. 533-542, Berlin, Germany, 2006. 